QUARTERLY OF APPLIED MATHEMATICS

VOLUME LXIV, NUMBER 1

MARCH 2006, PAGES 29-39

S $0033-569 \mathrm{X}(06) 00968-4$

Article electronically published on January 24, 2006

\title{
THE EXPONENTIAL DECAY OF GLOBAL SOLUTIONS TO THE GENERALIZED LANDAU EQUATION NEAR MAXWELLIANS
}

\author{
BY
}

HONGJUN YU

School of Mathematical Sciences, South China University of Technology, Guangzhou 510640, People's Republic of China and Academy of Mathematics and Systems Science, CAS, Beijing 100080, People's Republic of China

Abstract. Global-in-time classical solutions near Maxwellians are constructed for the generalized Landau equation in a periodic box for $\gamma \geq-2$. The exponential decay of such a solution is also obtained.

1. Introduction. In this paper, we consider the following generalized Landau equation

$$
\left[\partial_{t}+v \cdot \nabla_{x}\right] F=Q[F, F], \quad F(0, x, v)=F_{0}(x, v)
$$

where $F(t, x, v)$ is the spatially periodic distribution function for the particles at time $t \geq 0$, with spatial coordinates $x=\left(x_{1}, x_{2}, x_{3}\right) \in[-\pi, \pi]^{3}=T^{3}$ and velocity $v=$ $\left(v_{1}, v_{2}, v_{3}\right) \in R^{3}$. The collision between particles is given by the following generalized Landau operator,

$$
\begin{gathered}
Q[F, G]=\nabla_{v} \cdot\left\{\int_{R^{3}} \phi\left(v-v^{\prime}\right)\left[F\left(v^{\prime}\right) \nabla_{v} G(v)-G(v) \nabla_{v} F\left(v^{\prime}\right)\right] d v^{\prime}\right\} \\
=\partial_{i} \int_{R^{3}} \phi^{i j}\left(v-v^{\prime}\right)\left[F\left(v^{\prime}\right) \partial_{j} G(v)-G(v) \partial_{j} F\left(v^{\prime}\right)\right] d v^{\prime} .
\end{gathered}
$$

where $\phi^{i j}=\left\{\delta_{i j}-v_{i} v_{j} /|v|^{2}\right\}|v|^{\gamma+2}$, and $\gamma \geq-3$. The original Landau collision operator for the Coulombic interaction corresponds to the case $\gamma=-3$.

The conservation of the mass, momentum as well as energy, can be formulated as $(i=1,2,3)$

$$
\frac{d}{d t} \int_{T^{3} \times R^{3}} F(t)=\frac{d}{d t} \int_{T^{3} \times R^{3}} v_{i} F(t)=\frac{d}{d t} \int_{T^{3} \times R^{3}}|v|^{2} F(t) \equiv 0 .
$$

We study classical solutions for (1.1) near a global Maxwellian $\mu=e^{-|v|^{2}}$. We define the standard perturbation $f(t, x, v)$ to $\mu$ as $F=\mu+\mu^{1 / 2} f$. It is well known that

Received June 18, 2004.

2000 Mathematics Subject Classification. Primary 35Q99; Secondary 35A05.

Key words and phrases. Global classical solution, exponential decay, energy estimates.

E-mail address: yuhj@amss.ac.cn

(C)2006 Brown University 
$Q[\mu, \mu]=0$. By expanding $Q\left[\mu+\mu^{1 / 2} g_{1}, \mu+\mu^{1 / 2} g_{2}\right]$, we define

$$
Q\left[\mu+\mu^{1 / 2} g_{1}, \mu+\mu^{1 / 2} g_{2}\right] \equiv Q[\mu, \mu]+\mu^{1 / 2}\left\{K g_{1}+A g_{2}+\Gamma\left[g_{1}, g_{2}\right]\right\} .
$$

The system (1.1) for $f(t, x, v)$ becomes

$$
\left[\partial_{t}+v \cdot \nabla_{x}\right] f+L f=\Gamma[f, f], \quad f(0, x, v)=f_{0}(x, v),
$$

where $L=-A-K$. Notice that $A, K$ and $\Gamma$ are defined in the same way as in [5], namely, $\sigma^{i}=\phi^{i j} *\left[v_{j} \mu\right], \sigma^{i j}=\phi^{i j} * \mu$,

$$
\begin{gathered}
A g=\mu^{-1 / 2} \partial_{i}\left\{\mu^{1 / 2} \sigma^{i j}\left[\partial_{j} g+v_{j} g\right]\right\}, \quad K g=-\mu^{-1 / 2} \partial_{i}\left\{\mu\left[\phi^{i j} *\left\{\mu^{1 / 2}\left[\partial_{j} g+v_{j} g\right]\right\}\right]\right\}, \\
\Gamma\left[g_{1}, g_{2}\right]=\partial_{i}\left[\left\{\phi^{i j} *\left[\mu^{1 / 2} g_{1}\right]\right\} \partial_{j} g_{2}\right]-\left\{\phi^{i j} *\left[v_{i} \mu^{1 / 2} g_{1}\right]\right\} \partial_{j} g_{2} \\
-\partial_{i}\left[\left\{\phi^{i j} *\left[\mu^{1 / 2} \partial_{j} g_{1}\right]\right\} g_{2}\right]+\left\{\phi^{i j} *\left[v_{i} \mu^{1 / 2} \partial_{j} g_{1}\right]\right\} g_{2} .
\end{gathered}
$$

In the case when initially $F_{0}(x, v)$ has the same mass, momentum, and energy as Maxwellian $\mu$, we can rewrite the conservation laws as $(i=1,2,3)$,

$$
\int_{T^{3} \times R^{3}} f \mu^{1 / 2}=\int_{T^{3} \times R^{3}} v_{i} f \mu^{1 / 2}=\int_{T^{3} \times R^{3}}|v|^{2} f \mu^{1 / 2}=0 .
$$

We shall use $(\cdot, \cdot)$ to denote the standard $L^{2}$ inner product in $T^{3}, T^{3} \times R^{3}$ and $\|\cdot\|$ to denote the corresponding $L^{2}$ norms. Let the multi-indices $\alpha$ and $\beta$ be $\alpha=$ $\left[\alpha_{0}, \alpha_{1}, \alpha_{2}, \alpha_{3}\right]$ and $\beta=\left[\beta_{1}, \beta_{2}, \beta_{3}\right]$ with $|\alpha|=\sum_{k=0}^{3} \alpha_{k}$ and $|\beta|=\sum_{k=1}^{3} \beta_{k}$. We define $\partial_{\beta}^{\alpha} \equiv \partial_{t}^{\alpha_{0}} \partial_{x_{1}}^{\alpha_{1}} \partial_{x_{2}}^{\alpha_{2}} \partial_{x_{3}}^{\alpha_{3}} \partial_{v_{1}}^{\beta_{1}} \partial_{v_{2}}^{\beta_{2}} \partial_{v_{3}}^{\beta_{3}}$. If each component of $\beta$ is not greater than that of $\bar{\beta}$ 's, we denote it by $\beta \leq \bar{\beta}$. We define $\beta<\bar{\beta}$ if $\beta \leq \bar{\beta}$, and $|\beta|<|\bar{\beta}|$. We also $\operatorname{denote}\left(\frac{\beta}{\beta}\right)$ by $C_{\bar{\beta}}^{\beta}$.

We introduce a weight function of $v$ as $\omega=\omega(v)=[1+|v|]^{\gamma+2}$. We denote the weighted $L^{2}$ norm as $|g|_{2, \theta}^{2}=\int_{R^{3}} \omega^{2 \theta} g^{2} d v,\|g\|_{\theta}^{2}=\int_{R^{3} \times T^{3}} \omega^{2 \theta} g^{2} d x d v$, where $\|\cdot\|_{0}=\|\cdot\|$.

We define the weighted norm and the high order energy norm as

$$
\begin{gathered}
|g|_{\sigma, \theta}^{2}=\int_{R^{3}} \omega^{2 \theta}\left[\sigma^{i j} \partial_{i} g \partial_{j} g+\sigma^{i j} v_{i} v_{j} g^{2}\right] d v, \\
\|g\|_{\sigma, \theta}^{2}=\int_{R^{3} \times T^{3}} \omega^{2 \theta}\left[\sigma^{i j} \partial_{i} g \partial_{j} g+\sigma^{i j} v_{i} v_{j} g^{2}\right] d x d v, \\
E(f(t)) \equiv \frac{1}{2}\left|\|f \mid\|^{2}(t)+\int_{0}^{t}\|\| f\|\|_{\sigma}^{2}(s) d s,\right. \\
E\left(f_{0}\right)=E(f(0)) \equiv \sum_{|\alpha|+|\beta| \leq N}\left\|\partial_{\beta}^{\alpha} f_{0}\right\|^{2},
\end{gathered}
$$

where $|\cdot|_{\sigma, 0}=|\cdot|_{\sigma},\|\cdot\|_{\sigma, 0}=\|\cdot\|_{\sigma}$ and

$$
\left\||| f||(t) \equiv \sum_{|\alpha|+|\beta| \leq N}\right\| \partial_{\beta}^{\alpha} f(t)\|, \quad\||f|\left\|_{\sigma}(t) \equiv \sum_{|\alpha|+|\beta| \leq N}\right\| \partial_{\beta}^{\alpha} f(t) \|_{\sigma} .
$$

Throughout this article, $N \geq 8$. The main result is as follows:

Theorem 1.1. Let $\gamma \geq-2$. Assume that $f_{0}(x, v)$ satisfies $(1.3)$, and $F_{0}(x, v)=\mu+$ $\mu^{1 / 2} f_{0}(x, v) \geq 0$. There is an $C_{0}>0$ and $M>0$ such that if $E\left(f_{0}\right) \leq M$, then there exists a unique global solution $f(t, x, v)$ to (1.2) with $F(t, x, v)=\mu+\mu^{1 / 2} f(t, x, v) \geq 0$ 
and $\sup _{0 \leq s \leq \infty} E(f(s)) \leq C_{0} E\left(f_{0}\right)$. Moreover, there are constants $C_{1}>0$ and $\delta^{*}>0$ such that

$$
\||| f|| \mid(t) \leq C_{1} E^{1 / 2}\left(f_{0}\right) e^{-\delta^{*} t} .
$$

There have been some investigations about the dynamical problems of the Landau system [1], 2], 3], [5, 9], 10, [11, 12, 13, 14]. It is shown in [5 that the global classical solution of the Landau equation near Maxwellians with $\gamma \geq-3$ can be obtained by the energy method. Guo proves in 8 that under the hard sphere condition, the global in time solution of the Vlasov-Maxwell-Boltzmann system can be obtained. Desvillettes and Villani [2, 3] construct a global classical solution to the spatially homogeneous Landau equation with $0<\gamma \leq 1$, which converges towards equilibrium exponentially. It is shown in [10, 14] that the smooth solution of the spatially homogeneous Landau equation with $-3<\gamma<0$ converges to a global Maxwellian super-algebraically.

Motivated by an idea in [8], we establish a global in time classical solution to the generalized Landau equation near Maxwellians for $\gamma \geq-2$. And we also obtain the exponential decay of such a solution, that is, the global classical solution $F$ of (1.1) converges to a global Maxwellian $\mu$ in some Sobolev space exponentially. Compared to the previous work ([2], 3], [10], [14]]), it should be pointed out that the Landau equation discussed in the present paper is dependent on space variables, the global solutions here converge towards the equilibrium exponentially and the Landau collision operator concerned includes both hard potentials and soft potentials, i.e., $\gamma \geq-2$. To obtain the exponential decay of the global solution, we are not able to use the argument developed in [5, 6, 7] to construct the crucial positivity of the linearized Landau operator $L$. Instead, we have revised the methods in 8 to obtain it. It seems that global in time classical solutions to the Landau equation near Maxwellians for $-3 \leq \gamma<-2$ can also be established by this approach. But we could not obtain the exponential decay of the global classical solutions to the Landau equation near Maxwellians for $-3 \leq \gamma<-2$. Very recently, there has been new progress made by Strain and Guo [9] who obtain almost exponential decay of the global solutions for a large class of kinetic equations near Maxwellians, including the Landau equation for $-3 \leq \gamma<-2$.

2. The proof of Theorem 1.1. In this section we first give some lemmas which can be found in [5].

Lemma 2.1. Let $|\beta|>0,|\alpha|+|\beta| \leq N$. Then for small $\eta>0$, there exists $C>0$ and $C_{\eta}>0$ such that

$$
\begin{gathered}
-\left(\partial_{\beta}[A g], \partial_{\beta} g\right) \geq\left\|\partial_{\beta} g\right\|_{\sigma}^{2}-\eta \sum_{\left|\beta_{1}\right| \leq|\beta|}\left\|\partial_{\beta_{1}} g\right\|_{\sigma}^{2}-C_{\eta}\|\mu g\|^{2}, \\
\left|\left(\partial_{\beta}\left[K g_{1}\right], \partial_{\beta} g_{2}\right)\right| \leq\left\{\eta \sum_{\left|\beta_{1}\right| \leq|\beta|}\left\|\partial_{\beta_{1}} g_{1}\right\|_{\sigma}+C_{\eta}\left\|\mu g_{1}\right\|\right\}\left\|\partial_{\beta} g_{2}\right\|_{\sigma}, \\
\left(\partial_{\beta}^{\alpha} \Gamma\left[g_{1}, g_{2}\right], \partial_{\beta}^{\alpha} g_{3}\right) \leq C\left[\left\{\sum_{\left|\alpha_{1}\right|+\left|\beta_{1}\right| \leq N}\left\|\partial_{\beta_{1}}^{\alpha_{1}} g_{1}\right\|\right\}\left\{\sum_{\left|\alpha_{1}\right|+\left|\beta_{1}\right| \leq N}\left\|\partial_{\beta_{1}}^{\alpha_{1}} g_{2}\right\|_{\sigma}\right\}\right. \\
\left.+\left\{\sum_{\left|\alpha_{1}\right|+\left|\beta_{1}\right| \leq N}\left\|\partial_{\beta_{1}}^{\alpha_{1}} g_{1}\right\|_{\sigma}\right\}\left\{\sum_{\left|\alpha_{1}\right|+\left|\beta_{1}\right| \leq N}\left\|\partial_{\beta_{1}}^{\alpha_{1}} g_{2}\right\|\right\}\right]\left\|\partial_{\beta}^{\alpha} g_{3}\right\|_{\sigma} .
\end{gathered}
$$


Since $L \geq 0$ and $(L g, g)=0$ if and only if $g(v)=\left\{a+b \cdot v+c|v|^{2}\right\} \mu^{1 / 2}$ where $a, c \in R$ and $b \in R^{3}$, we denote the orthogonal basis for $\left\{1, v,|v|^{2}\right\} \mu^{1 / 2}$ in the same way as in 4. $\left\{e_{1}, e_{2}, e_{3}, e_{4}, e_{5}\right\}$ and we define a projection $P$ in $L^{2}\left(R^{3}\right)$ for any fixed $x$ as $P g(x, v)=\sum\left(g(x,),. e_{j}\right) e_{j}$.

Lemma 2.2. Let $\chi(v)$ be a smooth function so that $\left\{|\chi|+|\nabla \chi|+\left|\nabla^{2} \chi\right|\right\} \leq C \mu(v / 4)$, then

$$
\begin{gathered}
\left\|\int \partial^{\alpha} \Gamma\left[g_{1}, g_{2}\right] \chi d v\right\| \leq C\left\{\sum_{\left|\alpha_{1}\right| \leq N}\left\|\partial^{\alpha_{1}} g_{1}\right\|\right\}\left\{\sum_{\left|\alpha_{1}\right| \leq N}\left\|\partial^{\alpha_{1}} g_{2}\right\|_{\sigma}\right\}, \\
(L g, g) \geq \delta|\{I-P\} g|_{\sigma}^{2}, \\
|g|_{\sigma, \theta}^{2} \geq c\left|\omega^{\theta}[1+|v|]^{\frac{\gamma+2}{2}} g\right|_{2}^{2} .
\end{gathered}
$$

By a straightforward modification of the argument used in [5], we have the following local existence result for the Landau equation.

Lemma 2.3. For any sufficiently small $M_{0}>0$, there exists $T^{*}>0$ such that if $E\left(f_{0}\right) \leq$ $M_{0} / 2$, then there is a unique classical solution $f(t, x, v)$ to $(1.2)$ in $\left[0, T^{*}\right) \times T^{3} \times R^{3}$ such that $\sup _{0 \leq t \leq T^{*}} E(f(t)) \leq M_{0}$ and $E(f(t))$ is continuous over $\left[0, T^{*}\right)$. If $F_{0}(x, v)=$ $\mu+\mu^{1 / 2} f_{0} \geq 0$, then $F(t, x, v)=\mu+\mu^{1 / 2} f(t, x, v) \geq 0$. Furthermore, the conservation law (1.3) holds for all $0<t<T^{*}$ if they are valid initially at $t=0$.

We shall first establish the positivity of the linearized operator $L$ for every small amplitude solution $f(t, x, v)$ to $(1.2)$, and then prove the main result, Theorem 1.1. We know that $P$ is a projection from $L^{2}\left(R^{3}\right)$ to the null space of the linearized operator $L$. Thus, for any fixed $(t, x)$, a function $g(t, x, v)$ can be decomposed uniquely as

$$
g(t, x, v)=\{P g\}(t, x, v)+\{I-P\} g(t, x, v) .
$$

Split $f$ as $f(t, x, v)=\{P f\}(t, x, v)+\{I-P\} f(t, x, v)$ in the Landau equation (1.2). Thus, we have

$$
\left[\partial_{t}+v \cdot \nabla_{x}\right] P f=l(\{I-P\} f)+h(f),
$$

where

$$
l(\{I-P\} f) \equiv-\left[\partial_{t}+v \cdot \nabla_{x}+L\right]\{I-P\} f, \quad h(f) \equiv \Gamma[f, f] .
$$

Lemma 2.4. Let $\partial^{\alpha}=\partial_{t}^{\alpha_{0}} \partial_{x_{1}}^{\alpha_{1}} \partial_{x_{2}}^{\alpha_{2}} \partial_{x_{3}}^{\alpha_{3}}$. It can be shown that $\partial^{\alpha} P f=P \partial^{\alpha} f$. Furthermore, there exists $C>1$ such that for any $f \in C_{c}^{\infty}\left(R \times T^{3} \times R^{3}\right)$,

$$
\frac{1}{C}\left\|\partial^{\alpha} P f\right\|_{\sigma}^{2} \leq\left\|\partial^{\alpha} a\right\|^{2}+\left\|\partial^{\alpha} b\right\|^{2}+\left\|\partial^{\alpha} c\right\|^{2} \leq C\left\|\partial^{\alpha} P f\right\|^{2} .
$$

Proof. A direct computation implies $\partial^{\alpha} P f=P \partial^{\alpha} f$. We substitute $\|\cdot\|_{\sigma}$ with $P \partial^{\alpha} f=$ $\partial^{\alpha} a(t, x) \mu^{1 / 2}+\partial^{\alpha} b(t, x) \cdot v \mu^{1 / 2}+\partial^{\alpha} c(t, x)|v|^{2} \mu^{1 / 2}$. Using $\sigma^{i j} \leq C[1+|v|]^{\gamma+2}$ and the exponential decay of $e_{j}$, we can obtain the first half of (2.9) by a direct computation. The second half of (2.9) can be obtained by the fact that $\left|\partial^{\alpha} a\right|^{2}+\left|\partial^{\alpha} b\right|^{2}+\left|\partial^{\alpha} c\right|^{2}$ is bounded by $C \int\left|\partial^{\alpha} P f\right|^{2} d v$ for any $(t, x)$, since $a, b$ and $c$ are the coefficients of a basis to the null space of $L$. We then deduce (2.9) by a further integration over $x$. 
We now derive the macroscopic equations for $P f$ 's coefficients, $a, b$ and $c$. Recalling equation (2.7) and (2.8), we further use $P f=a(t, x) \mu^{1 / 2}+\sum_{j=1}^{3} \partial^{\alpha} b_{j}(t, x) v_{j} \mu^{1 / 2}+$ $\partial^{\alpha} c(t, x)|v|^{2} \mu^{1 / 2}$ to expand the entries of the left-hand side of (2.7) as

$$
\sum_{i}\left[v_{i} \partial^{i} c|v|^{2}+\left[\partial^{0} c+\partial^{i} b_{i}\right] v_{i}^{2}+\sum_{j>i}\left[\partial^{i} b_{j}+\partial^{j} b_{i}\right] v_{i} v_{j}+\left[\partial^{0} b_{i}+\partial^{i} a\right] v_{i}+\partial^{0} a\right] \mu^{1 / 2},
$$

where $\partial^{0}=\partial_{t}, \partial^{j}=\partial_{x_{j}}$ and $\partial^{i}=\partial_{x_{i}}$. This is an expansion to the left-hand side of (2.7), for fixed $(t, x)$, with respect to the basis of $\mu^{1 / 2}, v_{i} \mu^{1 / 2}, v_{i}^{2} \mu^{1 / 2}, v_{i} v_{j} \mu^{1 / 2}$ and $|v|^{2} v_{i} \mu^{1 / 2}$ where $1 \leq i \neq j \leq 3$. We denote an orthogonal basis for this 13-dimensional space by $\epsilon_{j}$, $1 \leq j \leq 13$ as in [4]. Expand the right-hand side of (2.7) with respect to the same basis, and compare with their coefficients on both sides. Then we have

$$
\begin{aligned}
& \text { (1) } \nabla_{x} c=l_{c}+h_{c}, \\
& \text { (2) } \partial^{0} c+\partial^{i} b_{i}=l_{i}+h_{i}, \\
& \text { (3) } \partial^{0} a=l_{a}+h_{a}, \\
& \text { (4) } \partial^{i} b_{j}+\partial^{j} b_{i}=l_{i j}+h_{i j}, \quad i \neq j, \\
& \text { (5) } \partial^{0} b_{i}+\partial^{i} a=l_{b i}+h_{b i},
\end{aligned}
$$

where $\partial^{0}=\partial_{t}$ and $\partial^{j}=\partial_{x_{j}}$. Here $l_{c}(t, x), l_{i}(t, x), l_{i j}(t, x), l_{b i}(t, x)$ and $l_{a}(t, x)$ are the corresponding coefficients of such an expansion of the linear term $l(\{I-P\} f)$, and $h_{c}(t, x), h_{i}(t, x), h_{i j}(t, x), h_{b i}(t, x)$ and $h_{a}(t, x)$ are the corresponding coefficients of the same expansion of the higher order term $h(f)$. Let

$$
\left[\mu^{1 / 2}, v_{i} \mu^{1 / 2}, v_{i}^{2} \mu^{1 / 2}, v_{i} v_{j} \mu^{1 / 2},|v|^{2} v_{i} \mu^{1 / 2}\right] A_{13 \times 13}=\left[\epsilon_{j}^{*}\right],
$$

with $\operatorname{det} A \neq 0$. We know that for any fixed $(t, x), l_{c}(t, x), l_{i}(t, x), l_{i j}(t, x), l_{b i}(t, x)$ and $l_{a}(t, x)$, which are the coefficients of the projection of $\{I-P\} f$, we have the form

$$
\sum_{i, n=1}^{13} \lambda^{i j} \lambda^{i n} \int_{R^{3}} l(\{I-P\} f) \cdot \epsilon_{j}(v) d v .
$$

The same is true after we take $\partial^{\alpha}$. Let $|\alpha| \leq N-1$. By (2.8), we have that

$$
\begin{aligned}
& \left\|\int\left(-\left[\partial_{t}+v \cdot \nabla_{x}\right]\{I-P\} \partial^{\alpha} f\right) \cdot \epsilon_{n}(v) d v\right\|^{2} \\
& \quad \leq \int\left|\epsilon_{n}(v)\right| d v \times \int_{R^{3} \times T^{3}}\left|\epsilon_{n}(v)\right|\left(\left|\{I-P\} \partial^{0} \partial^{\alpha} f\right|^{2}+|v|^{2}\left|\{I-P\} \nabla_{x} \partial^{\alpha} f\right|^{2}\right) d x d v \\
& \quad \leq C\left[\left\|\{I-P\} \partial^{0} \partial^{\alpha} f\right\|+\left\|\{I-P\} \nabla_{x} \partial^{\alpha} f\right\|\right]^{2}, \\
& \int L\{I-P\} \partial^{\alpha} f \epsilon_{n}(v) d v=\int(-A-K)\{I-P\} \partial^{\alpha} f \epsilon_{n}(v) d v .
\end{aligned}
$$

Recalling the expressions of $A, K$ and $\epsilon_{n}$, integration by parts and the Schwartz inequality will result in

$$
\left\|\int L\{I-P\} \partial^{\alpha} f \epsilon_{n}(v) d v\right\|^{2} \leq C\left\|\{I-P\} \partial^{\alpha} f\right\|^{2} .
$$

Here $\partial^{0}=\partial_{t}$, and we have also used the facts $\partial^{\alpha}\{I-P\} f=\{I-P\} \partial^{\alpha} f$ and the exponential decay of $\epsilon_{n}(v)$. Thus, we have

$$
\sum_{|\alpha| \leq N-1}\left\{\left\|\partial^{\alpha} l_{c}\right\|+\left\|\partial^{\alpha} l_{i}\right\|+\left\|\partial^{\alpha} l_{i j}\right\|+\left\|\partial^{\alpha} l_{b i}\right\|+\left\|\partial^{\alpha} l_{a}\right\|\right\} \leq C \sum_{|\alpha| \leq N}\left\|\{I-P\} \partial^{\alpha} f\right\| .
$$


Notice that $h(f)=\Gamma[f, f]$ and $\partial^{\alpha} h_{c}, \partial^{\alpha} h_{i}, \partial^{\alpha} h_{i j}, \partial^{\alpha} h_{b i}, \partial^{\alpha} h_{a}$ are of the form

$$
\sum_{i, n=1}^{13} \lambda^{i j} \lambda^{i n} \int_{R^{3}} \partial^{\alpha} \Gamma[f, f] \cdot \epsilon_{j}(v) d v,
$$

where $\lambda^{i j}$ and $\lambda^{i n}$ are the entries of the matrix $A$. If $|\alpha| \leq N$ and $\sum_{|\alpha| \leq N}\left\|\partial^{\alpha} f(t)\right\|^{2} \leq M_{0}$ for some $M_{0}>0$, then we can apply (2.4) in Lemma 2.2 to get

$$
\begin{aligned}
& \left\|\int \partial^{\alpha} \Gamma[f, f] \cdot \epsilon_{n}(v) d v\right\| \leq \sum C_{\alpha}^{\alpha_{1}}\left\|\int \Gamma\left[\partial^{\alpha_{1}} f, \partial^{\alpha-\alpha_{1}} f\right] \cdot \epsilon_{n}(v) d v\right\| \\
& \leq C\left\{\sum_{\left|\alpha_{1}\right| \leq N}\left\|\partial^{\alpha_{1}} f\right\|\right\}\left\{\sum_{\left|\alpha_{1}\right| \leq N}\left\|\partial^{\alpha_{1}} f\right\|_{\sigma}\right\} \leq C M_{0}^{1 / 2} \sum_{|\alpha| \leq N}\left\|\partial^{\alpha} f\right\|_{\sigma} .
\end{aligned}
$$

Thus, we have

$$
\sum_{|\alpha| \leq N}\left\{\left\|\partial^{\alpha} h_{c}\right\|+\left\|\partial^{\alpha} h_{i}\right\|+\left\|\partial^{\alpha} h_{i j}\right\|+\left\|\partial^{\alpha} h_{b i}\right\|+\left\|\partial^{\alpha} h_{a}\right\|\right\} \leq C M_{0}^{1 / 2} \sum_{|\alpha| \leq N}\left\|\partial^{\alpha} f\right\|_{\sigma} .
$$

Theorem 2.5. Let $f(t, x, v)$ be a classical solution to (1.2) satisfying (1.3). There exists $M_{0}>0$ and $\delta_{0}=\delta_{0}\left(M_{0}\right)>0$ such that if

$$
\sum_{|\alpha| \leq N}\left\|\partial^{\alpha} f(t)\right\|^{2} \leq M_{0}
$$

then

$$
\sum_{|\alpha| \leq N}\left(L \partial^{\alpha} f(s), \partial^{\alpha} f(s)\right) \geq \delta_{0} \sum_{|\alpha| \leq N}\left\|\partial^{\alpha} f(s)\right\|_{\sigma}^{2} .
$$

Proof. Recall that from (2.5) in Lemma 2.2

$$
\left(L \partial^{\alpha} f, \partial^{\alpha} f\right) \geq \delta\left\|\{I-P\} \partial^{\alpha} f\right\|_{\sigma}^{2} .
$$

It thus suffices to show that if (2.13) is valid for some small $M_{0}>0$, then there is a constant $C>0$ such that

$$
\sum_{|\alpha| \leq N}\left\|P \partial^{\alpha} f(t)\right\|_{\sigma} \leq C \sum_{|\alpha| \leq N}\left\|\{I-P\} \partial^{\alpha} f(t)\right\|_{\sigma} .
$$

We notice that by Lemma 2.4,

$$
\sum_{|\alpha| \leq N}\left\|P \partial^{\alpha} f(t)\right\|_{\sigma} \leq C \sum_{|\alpha| \leq N}\left\{\left\|\partial^{\alpha} a\right\|+\left\|\partial^{\alpha} b\right\|+\left\|\partial^{\alpha} c\right\|\right\} .
$$

Thus, we only need to prove that

$$
\sum_{|\alpha| \leq N}\left\{\left\|\partial^{\alpha} a\right\|+\left\|\partial^{\alpha} b\right\|+\left\|\partial^{\alpha} c\right\|\right\} \leq C \sum_{|\alpha| \leq N}\left\|\{I-P\} \partial^{\alpha} f(t)\right\|_{\sigma} .
$$


We first estimate $\nabla \partial^{\alpha} b$. Let $|\alpha| \leq N-1$. We take $\partial^{j}$ of (2) and (4) to get

$$
\begin{gathered}
\triangle \partial^{\alpha} b_{i}=\sum_{j} \partial^{j j} \partial^{\alpha} b_{i}=\sum_{j \neq i} \partial^{j j} \partial^{\alpha} b_{i}+\partial^{i i} \partial^{\alpha} b_{i}=\sum_{j \neq i}\left[-\partial^{j i} \partial^{\alpha} b_{j}+\partial^{j} \partial^{\alpha} l_{i j}+\partial^{j} \partial^{\alpha} h_{i j}\right] \\
+\left[\partial^{i} \partial^{\alpha} l_{i}+\partial^{i} \partial^{\alpha} h_{i}-\partial^{0} \partial^{i} \partial^{\alpha} c\right]=\sum_{j \neq i}\left[\partial^{0} \partial^{i} \partial^{\alpha} c-\partial^{i} \partial^{\alpha} l_{j}-\partial^{i} \partial^{\alpha} h_{j}\right]-\partial^{0} \partial^{i} \partial^{\alpha} c \\
+\sum_{j \neq i}\left[\partial^{j} l_{i j}+\partial^{j} h_{i j}\right]+\partial^{i} \partial^{\alpha} l_{i}+\partial^{i} \partial^{\alpha} h_{i}=\partial^{0} \partial^{i} \partial^{\alpha} c \\
-\sum_{i \neq j}\left[\partial^{i} \partial^{\alpha} l_{j}+\partial^{i} \partial^{\alpha} h_{j}-\partial^{j} \partial^{\alpha} l_{i j}-\partial^{j} \partial^{\alpha} h_{i j}\right]+\partial^{i} \partial^{\alpha} l_{i}+\partial^{i} \partial^{\alpha} h_{i} \\
=-\partial^{i i} \partial^{\alpha} b_{i}+2\left[\partial^{i} \partial^{\alpha} l_{i}+\partial^{i} \partial^{\alpha} h_{i}\right]-\sum_{i \neq j}\left[\partial^{i} \partial^{\alpha} l_{j}+\partial^{i} \partial^{\alpha} h_{j}-\partial^{j} \partial^{\alpha} l_{i j}-\partial^{j} \partial^{\alpha} h_{i j}\right] .
\end{gathered}
$$

We multiply with $\partial^{\alpha} b_{i}$ to get

$$
\begin{aligned}
&\left\|\nabla \partial^{\alpha} b_{i}\right\| \leq C \sum_{|\alpha| \leq N-1}\left\{\left\|\partial^{\alpha} l_{i}\right\|+\left\|\partial^{\alpha} h_{i}\right\|+\left\|\partial^{\alpha} l_{j}\right\|+\left\|\partial^{\alpha} h_{j}\right\|+\left\|\partial^{\alpha} l_{i j}\right\|+\left\|\partial^{\alpha} h_{i j}\right\|\right\} \\
& \leq C M_{0}^{1 / 2} \sum_{|\alpha| \leq N}\left\|\partial^{\alpha} f\right\|_{\sigma}+C \sum_{|\alpha| \leq N}\left\|\{I-P\} \partial^{\alpha} f\right\|
\end{aligned}
$$

where we have used (2.11) and (2.12). We will leave the proof of the purely temporal derivatives of $\partial^{\alpha} b_{i}(t, x)$ with $\alpha=\left[\alpha_{0}, 0,0,0\right]$ and $|\alpha| \leq N$ to the end.

Next we will estimate the derivatives of $c(t, x)$. From (1) and (2), we have

$$
\left\|\partial^{0} \partial^{\alpha} c\right\| \leq\left\|\partial^{i} \partial^{\alpha} b_{i}\right\|+\left\|\partial^{\alpha} l_{i}\right\|+\left\|\partial^{\alpha} h_{i}\right\|, \quad\left\|\nabla \partial^{\alpha} c\right\| \leq\left\|\partial^{\alpha} h_{c}\right\|+\left\|\partial^{\alpha} l_{c}\right\| .
$$

Thus, for $|\alpha| \leq N-1$, we have, from (2.11), (2.12) and (2.15), that

$$
\left\|\partial^{0} \partial^{\alpha} c\right\|+\left\|\nabla \partial^{\alpha} c\right\| \leq C M_{0}^{1 / 2} \sum_{|\alpha| \leq N}\left\|\partial^{\alpha} f\right\|_{\sigma}+C \sum_{|\alpha| \leq N}\left\|\{I-P\} \partial^{\alpha} f\right\| .
$$

From the Poincaré inequality, we easily see that $\|c\| \leq C\left[\|\nabla c\|+\left|\int c d x\right|\right]$. From the conservation laws in (1.3), we know that $\int_{T^{3}} b d x=0$ and

$$
\left|\int_{T^{3}} a d x\right|+\left|\int_{T^{3}} c d x\right|=0 .
$$

Thus, the term $\|c\|$ is controlled by the right-hand side of (2.16).

Now we consider $a(t, x)$. Let $|\alpha| \leq N-1$. By (3), we have

$$
\left\|\partial^{0} \partial^{\alpha} a\right\| \leq\left\|\partial^{\alpha} l_{a}\right\|+\left\|\partial^{\alpha} h_{a}\right\| .
$$

By (2.11) and (2.12), for $|\alpha| \leq N-1,\left\|\partial^{0} \partial^{\alpha} a\right\|$ is bounded by the right-hand side of (2.16). We now consider the spatial derivatives of $a(t, x)$. Let $|\alpha| \leq N-1$ and $\alpha=\left[0, \alpha_{1}, \alpha_{2}, \alpha_{3}\right] \neq 0$. By taking $\partial^{i}$ of (5) and summing over $i$, we get

$$
-\triangle \partial^{\alpha} a=\nabla \cdot \partial^{0} \partial^{\alpha} b-\sum_{i} \partial^{i} \partial^{\alpha}\left[l_{b i}+h_{b i}\right]
$$

Multiplying the above equation with $\partial^{\alpha} a$ and integrating over $T^{3}$, we get

$$
\left\|\nabla \partial^{\alpha} a\right\| \leq\left\|\partial^{0} \partial^{\alpha} b\right\|+\sum_{i}\left[\left\|\partial^{\alpha}\left[l_{b i}+h_{b i}\right]\right\|\right]
$$


It is clear that the right-hand side of (2.18) is bounded by the right-hand side of (2.16). Furthermore, by the the Poincaré inequality, we easily know $\|a\| \leq C\left[\|\nabla a\|+\left|\int a d x\right|\right]$. Thus, the right-hand side of it is bounded by the right-hand side of (2.16). We thus complete the estimate for $a(t, x)$.

Finally, we estimate the purely temporal derivatives of $b(t, x)$ and $\partial^{\alpha} b(t, x)$ with $\alpha=$ $\left[\alpha_{0}, 0,0,0\right]$. If $|\alpha| \leq 2$, we use the Poincaré inequality to get

$$
\left\|\partial^{\alpha} b_{i}\right\| \leq C\left\|\nabla \partial^{\alpha} b_{i}\right\|+\left|\partial^{\alpha} \int b_{i}(t, x) d x\right| .
$$

By (2.15) and $\int b_{i}(t, x) d x=0$, it suffices to estimate the term $\partial^{\alpha} b_{i}$. For the higher purely temporal derivative $\partial^{\alpha} b(t, x)$ with $|\alpha| \geq 3$, we take $\partial^{\alpha-1}$ of (5) to get

$$
\left\|\partial^{\alpha} b_{i}\right\|=\left\|-\partial^{i} \partial^{\alpha-1} a+\partial^{\alpha-1}\left[l_{b i}+h_{b i}\right]\right\| \leq\left\|\partial^{i} \partial^{\alpha-1} a\right\|+\left\|\partial^{\alpha-1} l_{b i}\right\|+\left\|\partial^{\alpha-1} h_{b i}\right\| .
$$

By (2.18) and (2.11), (2.12), we easily know that the right-hand side of the above inequality is bounded by the right-hand side of (2.16).

Therefore, we have, by the above estimates, that

$$
\sum_{|\alpha| \leq N}\left\{\left\|\partial^{\alpha} a\right\|+\left\|\partial^{\alpha} b\right\|+\left\|\partial^{\alpha} c\right\|\right\} \leq C M_{0}^{1 / 2} \sum_{|\alpha| \leq N}\left\|\partial^{\alpha} f\right\|_{\sigma}+C \sum_{|\alpha| \leq N}\left\|\{I-P\} \partial^{\alpha} f\right\| .
$$

The first term of the right-hand side of the above inequality can be neglected for $M_{0}$ small. This is because we have by Lemma 2.4 that

$$
\begin{gathered}
\left\|\partial^{\alpha} f\right\|_{\sigma}^{2} \leq\left\|\{I-P\} \partial^{\alpha} f\right\|_{\sigma}^{2}+\left\|P \partial^{\alpha} f\right\|_{\sigma}^{2} \\
\leq\left\|\{I-P\} \partial^{\alpha} f\right\|_{\sigma}^{2}+C\left[\left\|\partial^{\alpha} a\right\|+\left\|\partial^{\alpha} b\right\|+\left\|\partial^{\alpha} c\right\|\right]^{2} .
\end{gathered}
$$

By (2.6) with $\gamma \geq-2$, we know that (2.14) holds.

In the following we extend the local in time solution in Lemma 2.3 to the global in time solution. We need to prove the following theorem.

Theorem 2.6. Let $f(t, x, v)$ be the unique solution constructed in Lemma 2.3 which satisfies the conservation law (1.3). Let the small amplitude (2.13) be valid. Then, for any given $0 \leq m \leq N$ and $|\beta| \leq m$, there are constants $C_{|\beta|}>0, C_{m}^{*}>0$ and $\delta_{m}>0$ such that

$$
\sum_{|\beta| \leq m,|\alpha|+|\beta| \leq N}\left[C_{|\beta|} \frac{d}{d t}\left\|\partial_{\beta}^{\alpha} f(t)\right\|^{2}+\delta_{m}\left\|\partial_{\beta}^{\alpha} f(t)\right\|_{\sigma}^{2}\right] \leq C_{m}^{*} E^{1 / 2}(f(t))\|\| f\|\|_{\sigma}^{2}(t) .
$$

Proof. We use an induction over $m$, the order of the $v$-derivatives. For $m=0$, by taking the pure $\partial^{\alpha}$ of (1.2), we obtain

$$
\left[\partial_{t}+v \cdot \nabla+L\right] \partial^{\alpha} f=\partial^{\alpha} \Gamma[f, f] .
$$

Multiplying (2.20) by $\partial^{\alpha} f$ and integrating over $T^{3} \times R^{3}$, we obtain, by Theorem 2.5 and (2.3) in Lemma 2.1 with $g_{1}=g_{2}=g_{3}=f$, that

$$
\sum_{|\alpha| \leq N}\left[\frac{1}{2} \frac{d}{d t}\left\|\partial^{\alpha} f(t)\right\|^{2}+\delta_{0}\left\|\partial^{\alpha} f(t)\right\|_{\sigma}^{2}\right] \leq C E^{1 / 2}(f(t))\|\| f \mid \|_{\sigma}^{2}(t) .
$$

This concludes the case for $m=0$ with $C_{0}=1 / 2$ and $C_{0}^{*}=C$. 
Now assume the theorem is valid for $m$. For $|\beta|=m+1$, taking $\partial_{\beta}^{\alpha}(|\beta| \neq 0)$ of $(1.2)$, we obtain,

$$
\left[\partial_{t}+v \cdot \nabla_{x}\right] \partial_{\beta}^{\alpha} f+\partial_{\beta} L\left[\partial^{\alpha} f\right]+\sum_{\beta_{1} \neq 0} \partial_{\beta_{1}} v \cdot \nabla_{x} \partial_{\beta-\beta_{1}}^{\alpha} f=\partial_{\beta}^{\alpha} \Gamma[f, f] .
$$

For any $\eta>0$, applying Lemma 2.1 and $\|\mu g\| \leq C\|g\|_{\sigma}$, and then integrating over $T^{3}$, we deduce

$$
\begin{gathered}
-\left(\partial_{\beta} A\left[\partial^{\alpha} f\right], \partial_{\beta}^{\alpha} f\right) \geq\left\|\partial_{\beta}^{\alpha} f\right\|_{\sigma}^{2}-\eta \sum_{\left|\beta_{1}\right| \leq|\beta|}\left\|\partial_{\beta_{1}}^{\alpha} f\right\|_{\sigma}^{2}-C_{\eta}\left\|\partial^{\alpha} f\right\|_{\sigma}^{2}, \\
-\left(\partial_{\beta} K\left[\partial^{\alpha} f\right], \partial_{\beta}^{\alpha} f\right) \geq-\left\{\eta \sum_{\left|\beta_{1}\right| \leq|\beta|}\left\|\partial_{\beta_{1}}^{\alpha} f\right\|_{\sigma}+C_{\eta}\left\|\mu \partial^{\alpha} f\right\|\right\}\left\|\partial_{\beta}^{\alpha} f\right\|_{\sigma} \\
\geq-\eta \sum_{\left|\beta_{1}\right| \leq|\beta|}\left\|\partial_{\beta_{1}}^{\alpha} f\right\|_{\sigma}^{2}-\eta\left\|\partial_{\beta}^{\alpha} f\right\|_{\sigma}^{2}-C_{\eta}\left\|\partial^{\alpha} f\right\|_{\sigma}^{2} .
\end{gathered}
$$

Thus, we have, for any $\eta>0$,

$$
\left(\partial_{\beta} L\left[\partial^{\alpha} f\right], \partial_{\beta}^{\alpha} f\right) \geq\left\|\partial_{\beta}^{\alpha} f\right\|_{\sigma}^{2}-\eta \sum_{\left|\beta^{\prime}\right| \leq\left|\beta^{\prime}\right|}\left\|\partial_{\beta^{\prime}}^{\alpha} f\right\|_{\sigma}^{2}-C_{\eta}\left\|\partial^{\alpha} f\right\|_{\sigma}^{2} .
$$

For any $\eta>0$, we have

$$
\sum_{\beta_{1} \neq 0}\left(\partial_{\beta_{1}} v \cdot \nabla_{x} \partial_{\beta-\beta_{1}}^{\alpha} f, \partial_{\beta}^{\alpha} f\right) \leq \eta\left\|\partial_{\beta}^{\alpha} f\right\|^{2}+C_{\eta} \sum_{\left|\beta_{1}\right|=1}\left\|\nabla_{x} \partial_{\beta-\beta_{1}}^{\alpha} f\right\|^{2} .
$$

By (2.3), we easily see that

$$
\left(\partial_{\beta}^{\alpha} \Gamma[f, f], \partial_{\beta}^{\alpha} f\right) \leq C E^{1 / 2}(f(t)) \mid\|f\| \|_{\sigma}^{2}(t) .
$$

We thus have, by collecting terms and summing over $|\beta|=m+1$ and $|\alpha|+|\beta| \leq N$,

$$
\begin{aligned}
& \sum_{|\beta|=m+1,|\alpha|+|\beta| \leq N}\left[\frac{1}{2} \frac{d}{d t}\left\|\partial_{\beta}^{\alpha} f(t)\right\|^{2}+\left\|\partial_{\beta}^{\alpha} f(t)\right\|_{\sigma}^{2}\right] \\
& \leq \sum_{|\beta|=m+1,|\alpha|+|\beta| \leq N}\left[\sum_{|\beta|=m+1} 2 \eta\left\|\partial_{\beta}^{\alpha} f\right\|_{\sigma}^{2}+3 C_{\eta} \sum_{|\beta| \leq m,|\alpha|+|\beta| \leq N}\left\|\partial_{\beta}^{\alpha} f\right\|^{2}\right. \\
& \quad+C E^{1 / 2}(f(t))\left|\|f \mid\|_{\sigma}^{2}(t)\right] \\
& \leq Z_{m+1}\left[\sum_{|\beta|=m+1,|\alpha|+|\beta| \leq N} 2 \eta\left\|\partial_{\beta}^{\alpha} f\right\|_{\sigma}^{2}+3 C_{\eta} \sum_{|\beta| \leq m,|\alpha|+|\beta| \leq N}\left\|\partial_{\beta}^{\alpha} f\right\|^{2}\right. \\
& \left.\quad+C E^{1 / 2}(f(t))|| f \mid \|_{\sigma}^{2}(t)\right],
\end{aligned}
$$

where $Z_{m+1}$ denotes the number of all possible $(\alpha, \beta)$ such that $|\beta| \leq m+1$ and $|\alpha|+|\beta| \leq$ $N$. Choose $\eta=\frac{1}{4 Z_{m+1}}$, then there is a constant $C\left(Z_{m+1}\right)>0$ such that

$$
\begin{gathered}
\sum_{|\beta|=m+1,|\alpha|+|\beta| \leq N}\left[\frac{1}{2} \frac{d}{d t}\left\|\partial_{\beta}^{\alpha} f(t)\right\|^{2}+\frac{1}{2}\left\|\partial_{\beta}^{\alpha} f(t)\right\|_{\sigma}^{2}\right] \\
\leq C\left(Z_{m+1}\right)\left[\sum_{|\beta| \leq m,|\alpha|+|\beta| \leq N}\left\|\partial_{\beta}^{\alpha} f\right\|_{\sigma}^{2}+E^{1 / 2}(f(t))\|\| f \mid \|_{\sigma}^{2}(t)\right] .
\end{gathered}
$$


We may assume $C\left(Z_{m+1}\right)>1$. We multiply $(2.22)$ by $\frac{\delta_{m}}{2 C\left(Z_{m+1}\right)}$ and add it to (2.19) for $|\beta| \leq m$ to get

$$
\begin{aligned}
& \sum_{|\beta|=m+1,|\alpha|+|\beta| \leq N}\left[\frac{\delta_{m}}{4 C\left(Z_{m+1}\right)} \frac{d}{d t}\left\|\partial_{\beta}^{\alpha} f(t)\right\|^{2}+\frac{\delta_{m}}{4 C\left(Z_{m+1}\right)}\left\|\partial_{\beta}^{\alpha} f(t)\right\|_{\sigma}^{2}\right] \\
& +\sum_{|\beta| \leq m,|\alpha|+|\beta| \leq N}\left[C_{|\beta|} \frac{d}{d t}\left\|\partial_{\beta}^{\alpha} f(t)\right\|^{2}+\delta_{m}\left\|\partial_{\beta}^{\alpha} f(t)\right\|_{\sigma}^{2}\right] \\
& \leq \frac{\delta_{m}}{2} \sum_{|\beta| \leq m,|\alpha|+|\beta| \leq N}\left\|\partial_{\beta}^{\alpha} f(t)\right\|_{\sigma}^{2}+\left[C_{m}^{*}+\frac{\delta_{m}}{2}\right] E^{1 / 2}(f(t))\left|\|f \mid\|_{\sigma}^{2}(t) .\right.
\end{aligned}
$$

It is clear the first term on the right-hand side is absorbed by the last term on the left. We thus conclude the theorem by letting

$$
C_{m+1}=\frac{\delta_{m}}{4 C\left(Z_{m+1}\right)}, \quad \delta_{m+1}=\frac{\delta_{m}}{4 C\left(Z_{m+1}\right)} \leq \frac{\delta_{m}}{2}, \quad C_{m+1}^{*}=C_{m}^{*}+\frac{\delta_{m}}{2} .
$$

Proof of Theorem 1.1. We first fix $M_{0} \leq 1$ such that both Lemma 2.3 and Theorem 2.6 are valid. For such a $M_{0}$, we let $m=N$ in (2.19) and define

$$
y(t) \equiv \sum_{|\alpha|+|\beta| \leq N}\left[C_{|\beta|} \mid\left\|\partial_{\beta}^{\alpha} f(t)\right\|^{2}\right] .
$$

We choose a constant $C_{1}>1$ such that for any $t \geq 0$,

$$
\frac{1}{C_{1}}\left[y(t)+\frac{\delta_{N}}{2} \int_{0}^{t} \mid\|f\|_{\sigma}^{2}(s)\right] d s \leq E(f(t)) \leq C_{1}\left[y(t)+\frac{\delta_{N}}{2} \int_{0}^{t}\||f|\|_{\sigma}^{2}(s) d s\right] .
$$

Recall constant $C_{N}^{*}$ in (2.19). We define $M \equiv \min \left\{\frac{\delta_{N}^{2}}{8 C_{N}^{* 2} C_{1}^{2}}, \frac{M_{0}}{2 C_{1}^{2}}\right\}$, and choose initial data so that $E\left(f_{0}\right) \leq M<M_{0}$. From Lemma 2.3, we may denote $T>0$ so that

$$
T=\sup _{t}\left\{t: E(f(t)) \leq 2 C_{1}^{2} M\right\}>0 .
$$

Notice that $E(f(t)) \leq 2 C_{1}^{2} M \leq M_{0}$ for $0 \leq t \leq T$, by the definition of $M$. Thus, the small amplitude assumption (2.13) is valid. We apply Theorem 2.6 and the definition of $M$ and $T$ to get, for $0 \leq t \leq T$, that

$$
y^{\prime}(t)+\delta_{N}\left|\left\|f \left|\left\|_{\sigma}^{2}(t) \leq C_{N}^{*} E^{1 / 2}(f(t))\left|\left\|f\left|\left\|_{\sigma}^{2}(t) \leq \frac{\delta_{N}}{2}\right\| f\right|\right\|_{\sigma}^{2}(t) .\right.\right.\right.\right.\right.
$$

Therefore, an integration over $0 \leq t \leq s<T$ yields

$$
\begin{aligned}
& E(f(s)) \leq C_{1}\left[y(s)+\frac{\delta_{N}}{2} \int_{0}^{s}\||f|\|_{\sigma}^{2}(\tau) d \tau\right] \\
& \leq C_{1} y(0) \leq C_{1}^{2} E(f(0)) \leq C_{1}^{2} M<2 C_{1}^{2} M .
\end{aligned}
$$

Since $E(f(s))$ is continuous in $s$, this implies $E(f(T)) \leq C_{1}^{2} M$ if $T<\infty$. Thus, we get a contradiction to the definition of $T$. Hence $T=\infty$. It is easily seen from the above inequality that such a global solution satisfies $E(f(t)) \leq C_{1}^{2} E\left(f_{0}\right)$ for all $t \geq 0$. By (2.24), we have for $t \geq 0$,

$$
y^{\prime}(t)+\frac{\delta_{N}}{2}|||f| \|_{\sigma}^{2}(t) \leq 0
$$


We easily know $C_{3}\left|\|f \mid\|(t) \leq y(t) \leq C_{2}\|\| f \|_{\sigma}(t)\right.$ by (2.24) and (2.6) with $\gamma \geq-2$. Thus, there is $C_{4}>0$ and $\delta^{*}>0$ such that

$$
\||| f|| \mid(t) \leq C_{4} E^{1 / 2}\left(f_{0}\right) e^{-\delta^{*} t} .
$$

Acknowledgements We would like to thank Professor Guo Yan and Dr. Strain for their kindness for sending us the paper $[9]$.

\section{REFERENCES}

[1] Degond P. and Lemou M., Dispersion relations for the linearized Fokker-Planck equation. Arch. Rat. Mech. Anal., 138 (2) (1989), 137-167. MR1463805 (99f:82051)

[2] Desvillettes L. and Villani C., On the spatially homogeneous Landau equation for hard potentials I. Existence, uniqueness and smoothness. Comm. P.D.E. 25(1-2), (2000), 179-259. MR.1737547 (2001c:82065)

[3] Desvillettes L. and Villani C., On the spatially homogeneous Landau equation for hard potentials II. H-Theorem and application. Comm. P.D.E. 25(1-2), (2000), 261-298. MR.1737548(2001c:82066)

[4] Glassey R., The Cauchy problem in kinetic theory. SIAM, Philadelphia, PA, 1996. MR1379589 (97i:82070)

[5] Guo Y., The Landau Equation in a periodic box. Comm. Math. Phys., 231 (2002), 391-434. MR.1946444(2004c:82121)

[6] Guo Y., Classical solutions to the Boltzmann equation for molecules with angular cutoff. Arch. Rat. Mech. Anal., 169 (2003), 305-353. MR2013332(2004i:82054)

[7] Guo Y., The Vlasov-Poisson-Boltzmann system near Maxwellians. Comm. Pure Appl. Math., 55 (2002), 1104-1135. MR 1908664 (2003b:82050)

[8] Guo Y., The Vlasov-Maxwell-Boltzmann system near Maxwellians. Invent. Math., 153 (2003), 593630. MR2000470 (2004m:82123)

[9] Strain R. and Guo Y., Almost exponential decay in kinetic equations. Preprint, 2004.

[10] Toscani, G. and Villani C., On the trend to equilibrium for some dissipative systems with slowly increasing a priori bounds. J. Statist. Phys., 98 (5-6) (2000), 1279-1309. MR.1751701 (2001g:82069)

[11] Yu, H.J., Global solution of the Vlasov-Poisson-Landau systems near Maxwellians with small amplitude., J. Partial Diff. Eqs. 17 (2)(2004), 173-192. MR2060788

[12] Villani C., On a new class of weak solutions to the spatial homogeneous Boltzmann and Landau equations. Arch. Rat. Mech. Anal., 143 (3) (1998), 237-307. MR1650006 (99j:82065)

[13] Villani C., On the Cauchy problem for Landau equation: Sequential stability, global existence. Adv. Diff. Eq. 1 (5) (1996), 793-816. MR392006 (97e:82048)

[14] Villani C., A survey of mathematical topics in kinetic theory. To appear in Handbook of Fluid Mechanics, S. Friedlander and D. Serre, Eds. 